KYUNGPOOK Math. J. 54(2014), 73-86

http://dx.doi.org/10.5666/KMJ.2014.54.1.73

\title{
Some Difference Paranormed Sequence Spaces over $n$-normed Spaces Defined by a Musielak-Orlicz Function
}

\author{
Kuldip RaJ*, Sunil K. Sharma and Amit Gupta \\ School of Mathematics Shri Mata Vaishno Devi University Katra-182320, JEK, In- \\ dia \\ e-mail : kuldeepraj68@rediffmail.com, sunilksharma42@yahoo.co.in \\ and guptaamit796@gmail.com
}

ABSTRACT. In the present paper we introduce difference paranormed sequence spaces $c_{0}\left(\mathcal{M}, \Delta_{m}^{n}, p, u,\|\cdot, \cdots, \cdot\|\right), c\left(\mathcal{M}, \Delta_{m}^{n}, p, u,\|\cdot, \cdots, \cdot\|\right)$ and $l_{\infty}\left(\mathcal{M}, \Delta_{m}^{n}, p, u,\|\cdot, \cdots, \cdot\|\right)$ defined by a Musielak-Orlicz function $\mathcal{M}=\left(M_{k}\right)$ over $n$-normed spaces. We also study some topological properties and some inclusion relations between these spaces.

\section{Introduction and Preliminaries}

Let $w, l_{\infty}, c$ and $c_{0}$ denote the spaces of all, bounded, convergent and null sequences $x=\left(x_{k}\right)$ with real or complex entries respectively. The zero sequence is denoted by $\theta=(0,0, \ldots)$. The notion of difference sequence spaces was introduced by Kızmaz [9], who studied the difference sequence spaces $l_{\infty}(\Delta), c(\Delta)$ and $c_{0}(\Delta)$. The notion was further generalized by Et and Çolak [4] by introducing the spaces $l_{\infty}\left(\Delta^{n}\right), c\left(\Delta^{n}\right)$ and $c_{o}\left(\Delta^{n}\right)$. Let $m, n$ be non-negative integers, then for $Z=l_{\infty}, c$ and $c_{0}$ we have sequence spaces,

$$
Z\left(\Delta_{m}^{n}\right)=\left\{x=\left(x_{k}\right) \in w:\left(\Delta_{m}^{n} x_{k}\right) \in Z\right\}
$$

where $\Delta_{m}^{n} x=\left(\Delta_{m}^{n} x_{k}\right)=\left(\Delta_{m}^{n-1} x_{k}-\Delta_{m}^{n-1} x_{k+m}\right)$ and $\Delta_{m}^{0} x_{k}=x_{k}$ for all $k \in \mathbb{N}$, which is equivalent to the following binomial representation

$$
\Delta_{m}^{n} x_{k}=\sum_{v=0}^{n}(-1)^{v}\left(\begin{array}{c}
n \\
v
\end{array}\right) x_{k+m v}
$$

Taking $m=n=1$, we get the spaces $l_{\infty}(\Delta), c(\Delta)$ and $c_{o}(\Delta)$ introduced and studied by Kizmaz [6].

Let $X$ be a linear metric space. A function $p: X \rightarrow \mathbb{R}$ is called paranorm, if

* Corresponding Author.

Received August 18, 2011; accepted August 22, 2012.

2000 Mathematics Subject Classification: 40A05, 46A45, 46E30.

Key words and phrases: Paranorm space, difference sequence spaces, Orlicz function, Musielak-Orlicz function, solid, monotone etc. 
1. $p(x) \geq 0$, for all $x \in X$;

2. $p(-x)=p(x)$, for all $x \in X$;

3. $p(x+y) \leq p(x)+p(y)$, for all $x, y \in X$;

4. if $\left(\sigma_{n}\right)$ is a sequence of scalars with $\sigma_{n} \rightarrow \sigma$ as $n \rightarrow \infty$ and $\left(x_{n}\right)$ is a sequence of vectors with $p\left(x_{n}-x\right) \rightarrow 0$ as $n \rightarrow \infty$, then $p\left(\sigma_{n} x_{n}-\sigma x\right) \rightarrow 0$ as $n \rightarrow \infty$.

A paranorm $p$ for which $p(x)=0$ implies $x=0$ is called total paranorm and the pair $(X, p)$ is called a total paranormed space. It is well known that the metric of any linear metric space is given by some total paranorm (see [18], Theorem 10.4.2, P-183). For more details about sequence spaces (see [1], [2], [3], [14], [15], [16], [17]) and references therein.

An Orlicz function $M:[0, \infty) \rightarrow[0, \infty)$ is a continuous and convex with $M(0)=0, M(x)>0$ for $x>0$ and $M(x) \longrightarrow \infty$ as $x \longrightarrow \infty$.

Lindenstrauss and Tzafriri [10] used the idea of Orlicz function to define the following sequence space,

$$
\ell_{M}=\left\{\left(x_{k}\right) \in w: \sum_{k=1}^{\infty} M\left(\frac{\left|x_{k}\right|}{\rho}\right)<\infty, \text { for some } \rho>0\right\}
$$

which is called as an Orlicz sequence space. Also $\ell_{M}$ is a Banach space with the norm

$$
\left\|\left(x_{k}\right)\right\|=\inf \left\{\rho>0: \sum_{k=1}^{\infty} M\left(\frac{\left|x_{k}\right|}{\rho}\right) \leq 1\right\} .
$$

Also, it was shown in [10] that every Orlicz sequence space $\ell_{M}$ contains a subspace isomorphic to $\ell_{p}(p \geq 1)$. An Orlicz function $M$ satisfies $\Delta_{2}-$ condition if and only if for any constant $L>1$ there exists a constant $K(L)$ such that $M(L u) \leq K(L) M(u)$ for all values of $u \geq 0$. An Orlicz function $M$ can always be represented in the following integral form

$$
M(x)=\int_{0}^{x} \eta(t) d t
$$

where $\eta$ is known as the kernel of $M$, is right differentiable for $t \geq 0, \eta(0)=0, \eta(t)>$ $0, \eta$ is non-decreasing and $\eta(t) \rightarrow \infty$ as $t \rightarrow \infty$.

A sequence $\mathcal{M}=\left(M_{k}\right)$ of Orlicz functions is called a Musielak-Orlicz function (see [11], [13]). A sequence $\mathcal{N}=\left(N_{k}\right)$ defined by

$$
N_{k}(v)=\sup \left\{|v| u-M_{k}(u): u \geq 0\right\}, k=1,2, \cdots
$$

is called the complementary function of a Musielak-Orlicz function $\mathcal{M}$. For a given Musielak-Orlicz function $\mathcal{M}$, the Musielak-Orlicz sequence space $t_{\mathcal{M}}$ and its subspace $h_{\mathcal{M}}$ are defined as follows

$$
t_{\mathcal{M}}=\left\{x \in w: I_{\mathcal{M}}(c x)<\infty \text { for some } c>0\right\},
$$




$$
h_{\mathcal{M}}=\left\{x \in w: I_{\mathcal{M}}(c x)<\infty \text { for all } c>0\right\},
$$

where $I_{\mathcal{M}}$ is a convex modular defined by

$$
I_{\mathcal{M}}(x)=\sum_{k=1}^{\infty} M_{k}\left(x_{k}\right), x=\left(x_{k}\right) \in t_{\mathcal{M}} .
$$

We consider $t_{\mathcal{M}}$ equipped with the Luxemburg norm

$$
\|x\|=\inf \left\{k>0: I_{\mathcal{M}}\left(\frac{x}{k}\right) \leq 1\right\}
$$

or equipped with the Orlicz norm

$$
\|x\|^{0}=\inf \left\{\frac{1}{k}\left(1+I_{\mathcal{M}}(k x)\right): k>0\right\} .
$$

The concept of 2-normed spaces was initially developed by Gähler [5] in the mid of 1960 's, while that of $n$-normed spaces one can see in Misiak[9]. Since then, many others have studied this concept and obtained various results, see Gunawan ([6], [7]) and Gunawan and Mashadi [8]. Let $n \in \mathbb{N}$ and $X$ be a linear space over the field $\mathbb{K}$, where $\mathbb{K}$ is the field of real or complex numbers of dimension $d$, where $d \geq n \geq 2$. A real valued function $\|\cdot, \cdots, \cdot\|$ on $X^{n}$ which satisfies the following four conditions:

1. $\left\|x_{1}, x_{2}, \cdots, x_{n}\right\|=0$ if and only if $x_{1}, x_{2}, \cdots, x_{n}$ are linearly dependent in $X$

2. $\left\|x_{1}, x_{2}, \cdots, x_{n}\right\|$ is invariant under permutation;

3. $\left\|\alpha x_{1}, x_{2}, \cdots, x_{n}\right\|=|\alpha|\left\|x_{1}, x_{2}, \cdots, x_{n}\right\|$ for any $\alpha \in \mathbb{K}$, and

4. $\left\|x+x^{\prime}, x_{2}, \cdots, x_{n}\right\| \leq\left\|x, x_{2}, \cdots, x_{n}\right\|+\left\|x^{\prime}, x_{2}, \cdots, x_{n}\right\|$

is called a $n$-norm on $X$ and the pair $(X,\|\cdot, \cdots, \cdot\|)$ is called a $n$-normed space over the field $\mathbb{K}$.

For example, we may take $X=\mathbb{R}^{n}$ being equipped with

the $n$-norm $\left\|x_{1}, x_{2}, \cdots, x_{n}\right\|_{E}=$ the volume of the $n$-dimensional parallelopiped spanned by the vectors $x_{1}, x_{2}, \cdots, x_{n}$ which may be given explicitly by the formula

$$
\left\|x_{1}, x_{2}, \cdots, x_{n}\right\|_{E}=\left|\operatorname{det}\left(x_{i j}\right)\right|,
$$

where $x_{i}=\left(x_{i 1}, x_{i 2}, \cdots, x_{i n}\right) \in \mathbb{R}^{n}$ for each $i=1,2, \cdots, n$ and script $E$ denotes the Euclidean norm. Let $(X,\|\cdot, \cdots, \cdot\|)$ be a $n$-normed space of dimension $d \geq n \geq 2$ and $\left\{a_{1}, a_{2}, \cdots, a_{n}\right\}$ be linearly independent set in $X$. Then the following function $\|\cdot, \cdots, \cdot\|_{\infty}$ on $X^{n-1}$ defined by

$$
\left\|x_{1}, x_{2}, \cdots, x_{n-1}\right\|_{\infty}=\max \left\{\left\|x_{1}, x_{2}, \cdots, x_{n-1}, a_{i}\right\|: i=1,2, \cdots, n\right\}
$$

defines an $(n-1)$-norm on $X$ with respect to $\left\{a_{1}, a_{2}, \cdots, a_{n}\right\}$. 
A sequence $\left(x_{k}\right)$ in a $n$-normed space $(X,\|\cdot, \cdots, \cdot\|)$ is said to converge to some $L \in X$ if

$$
\lim _{k \rightarrow \infty}\left\|x_{k}-L, z_{1}, \cdots, z_{n-1}\right\|=0 \text { for every } z_{1}, \cdots, z_{n-1} \in X .
$$

A sequence $\left(x_{k}\right)$ in a $n$-normed space $(X,\|\cdot, \cdots, \cdot\|)$ is said to be Cauchy if

$$
\lim _{k, p \rightarrow \infty}\left\|x_{k}-x_{p}, z_{1}, \cdots, z_{n-1}\right\|=0 \text { for every } z_{1}, \cdots, z_{n-1} \in X .
$$

If every Cauchy sequence in $X$ converges to some $L \in X$, then $X$ is said to be complete with respect to the $n$-norm. Any complete $n$-normed space is said to be $n$-Banach space.

Let $\mathcal{M}=\left(M_{k}\right)$ be a Musielak-Orlicz function, $p=\left(p_{k}\right)$ be a bounded sequence of positive real numbers and $u=\left(u_{k}\right)$ be a sequence of positive reals such that $u_{k} \neq 0$ for all $k$, then we define the following classes of sequences in the present paper:

$c_{0}\left(\mathcal{M}, \Delta_{m}^{n}, p, u,\|\cdot, \cdots, \cdot\|\right)=$

$$
\begin{array}{r}
\left\{x=\left(x_{k}\right) \in w: \lim _{k \rightarrow \infty} u_{k}\left[M_{k}\left(\left\|\frac{\Delta_{m}^{n} x_{k}}{\rho}, z_{1}, \cdots, z_{n-1}\right\|\right)\right]^{p_{k}}=0, \text { for some } \rho>0\right\}, \\
c\left(\mathcal{M}, \Delta_{m}^{n}, p, u,\|\cdot, \cdots, \cdot\|\right)=\left\{x=\left(x_{k}\right) \in w: \lim _{k \rightarrow \infty} u_{k}\left[M_{k}\left(\left\|\frac{\Delta_{m}^{n} x_{k}-L}{\rho}, z_{1}, \cdots, z_{n-1}\right\|\right)\right]^{p_{k}}\right. \\
=0, \text { for some } \rho>0 \text { and } L \in X\},
\end{array}
$$

and

$$
\begin{aligned}
& l_{\infty}\left(\mathcal{M}, \Delta_{m}^{n}, p, u,\|\cdot, \cdots, \cdot\|\right)= \\
& \left\{x=\left(x_{k}\right) \in w: \sup _{k \geq 1} u_{k}\left[M_{k}\left(\left\|\frac{\Delta_{m}^{n} x_{k}}{\rho}, z_{1}, \cdots, z_{n-1}\right\|\right)\right]^{p_{k}}<\infty, \text { for some } \rho>0\right\} .
\end{aligned}
$$

For $p_{k}=1$, for all $k$

$c_{0}\left(\mathcal{M}, \Delta_{m}^{n}, u,\|\cdot, \cdots, \cdot\|\right)=$

$$
\begin{array}{r}
\left\{x=\left(x_{k}\right) \in w: \lim _{k \rightarrow \infty} u_{k}\left[M_{k}\left(\left\|\frac{\Delta_{m}^{n} x_{k}}{\rho}, z_{1}, \cdots, z_{n-1}\right\|\right)\right]=0, \text { for some } \rho>0\right\}, \\
c\left(\mathcal{M}, \Delta_{m}^{n}, u,\|\cdot, \cdots, \cdot\|\right)=\left\{x=\left(x_{k}\right) \in w: \lim _{k \rightarrow \infty} u_{k}\left[M_{k}\left(\left\|\frac{\Delta_{m}^{n} x_{k}-L}{\rho}, z_{1}, \cdots, z_{n-1}\right\|\right)\right]\right. \\
=0, \text { for some } \rho>0 \text { and } L \in X\},
\end{array}
$$

and

$$
\begin{aligned}
& l_{\infty}\left(\mathcal{M}, \Delta_{m}^{n}, u,\|\cdot, \cdots, \cdot\|\right)= \\
& \quad\left\{x=\left(x_{k}\right) \in w: \sup _{k \geq 1} u_{k}\left[M_{k}\left(\left\|\frac{\Delta_{m}^{n} x_{k}}{\rho}, z_{1}, \cdots, z_{n-1}\right\|\right)\right]<\infty, \text { for some } \rho>0\right\} .
\end{aligned}
$$


For $\mathcal{M}(x)=x$, we have

$$
\begin{aligned}
& c_{0}\left(\Delta_{m}^{n}, p, u,\|\cdot, \cdots, \cdot\|\right)=\left\{x=\left(x_{k}\right) \in w: \lim _{k \rightarrow \infty} u_{k}\left(\left\|\frac{\Delta_{m}^{n} x_{k}}{\rho}, z_{1}, \cdots, z_{n-1}\right\|\right)^{p_{k}}=0,\right. \\
& \text { for some } \rho>0\}, \\
& c\left(\Delta_{m}^{n}, p, u,\|\cdot, \cdots, \cdot\|\right)=\left\{x=\left(x_{k}\right) \in w: \lim _{k \rightarrow \infty} u_{k}\left(\left\|\frac{\Delta_{m}^{n} x_{k}-L}{\rho}, z_{1}, \cdots, z_{n-1}\right\|\right)^{p_{k}}\right. \\
& =0, \text { for some } \rho>0 \text { and } L \in X\},
\end{aligned}
$$

and

$$
\begin{gathered}
l_{\infty}\left(\Delta_{m}^{n}, p, u,\|\cdot, \cdots, \cdot\|\right)=\left\{x=\left(x_{k}\right) \in w: \sup _{k \geq 1} u_{k}\left(\left\|\frac{\Delta_{m}^{n} x_{k}}{\rho}, z_{1}, \cdots, z_{n-1}\right\|\right)^{p_{k}}<\infty,\right. \\
\text { for some } \rho>0\} .
\end{gathered}
$$

The following inequality will be used throughout the paper. If $0 \leq p_{k} \leq \sup p_{k}=G$, $K=\max \left(1,2^{G-1}\right)$ then

$$
\left|a_{k}+b_{k}\right|^{p_{k}} \leq K\left\{\left|a_{k}\right|^{p_{k}}+\left|b_{k}\right|^{p_{k}}\right\}
$$

for all $k$ and $a_{k}, b_{k} \in \mathbb{C}$. Also $|a|^{p_{k}} \leq \max \left(1,|a|^{G}\right)$ for all $a \in \mathbb{C}$.

The aim of this paper is to study some difference sequence spaces in more general setting i.e. over $n$ - normed spaces defined by a Musielak-Orlicz function.

\section{Main Results}

In this section, we study some topological properties and inclusion relation between the spaces $c_{0}\left(\mathcal{M}, \Delta_{m}^{n}, p, u,\|\cdot, \cdots, \cdot\|\right), c\left(\mathcal{M}, \Delta_{m}^{n}, p, u,\|\cdot, \cdots, \cdot\|\right)$ and $l_{\infty}\left(\mathcal{M}, \Delta_{m}^{n}, p, u,\|\cdot, \cdots, \cdot\|\right)$.

Theorem 2.1. Let $\mathcal{M}=\left(M_{k}\right)$ be a Musielak-Orlicz function, $p=\left(p_{k}\right)$ be a bounded sequence of positive real numbers and $u=\left(u_{k}\right)$ be a sequence of strictly positive real numbers, then the classes of sequences $c_{0}\left(\mathcal{M}, \Delta_{m}^{n}, p, u,\|\cdot, \cdots, \cdot\|\right)$, $c\left(\mathcal{M}, \Delta_{m}^{n}, p, u,\|\cdot, \cdots, \cdot\|\right)$ and $l_{\infty}\left(\mathcal{M}, \Delta_{m}^{n}, p, u,\|\cdot, \cdots, \cdot\|\right)$ are linear spaces.

Proof. Let $x=\left(x_{k}\right), y=\left(y_{k}\right) \in c_{0}\left(\mathcal{M}, \Delta_{m}^{n}, p, u,\|\cdot, \cdots, \cdot\|\right)$ and $\alpha, \beta \in \mathbb{C}$. Then there exist positive numbers $\rho_{1}$ and $\rho_{2}$ such that

$$
\begin{gathered}
\lim _{k \rightarrow \infty} u_{k}\left[M_{k}\left(\left\|\frac{\Delta_{m}^{n} x_{k}}{\rho_{1}}, z_{1}, \cdots, z_{n-1}\right\|\right)\right]^{p_{k}}=0, \text { and } \\
\lim _{k \rightarrow \infty} u_{k}\left[M_{k}\left(\left\|\frac{\Delta_{m}^{n} y_{k}}{\rho_{2}}, z_{1}, \cdots, z_{n-1}\right\|\right)\right]^{p_{k}}=0 .
\end{gathered}
$$


Let $\rho_{3}=\max \left(2|\alpha| \rho_{1}, 2|\beta| \rho_{2}\right)$. Since $\mathcal{M}=\left(M_{k}\right)$ is non-decreasing convex function and so by using inequality (1.1), we have

$$
\begin{aligned}
\lim _{k \rightarrow \infty} u_{k} & {\left[M_{k}\left(\left\|\frac{\Delta_{m}^{n}\left(\alpha x_{k}+\beta y_{k}\right) \mid}{\rho_{3}}, z_{1}, \cdots, z_{n-1}\right\|\right)\right]^{p_{k}} } \\
\leq & \lim _{k \rightarrow \infty} u_{k}\left[M_{k}\left(\left\|\frac{\alpha \Delta_{m}^{n} x_{k}}{\rho_{3}}, z_{1}, \cdots, z_{n-1}\right\|+\left\|\frac{\beta \Delta_{m}^{n} y_{k}}{\rho_{3}}, z_{1}, \cdots, z_{n-1}\right\|\right)\right]^{p_{k}} \\
\leq & K \lim _{k \rightarrow \infty} \frac{1}{2^{p_{k}}} u_{k}\left[M_{k}\left(\left\|\frac{\Delta_{m}^{n} x_{k}}{\rho_{1}}, z_{1}, \cdots, z_{n-1}\right\|\right)\right]^{p_{k}} \\
& +K \lim _{k \rightarrow \infty} \frac{1}{2^{p_{k}}} u_{k}\left[M_{k}\left(\left\|\frac{\Delta_{m}^{n} y_{k}}{\rho_{2}}, z_{1}, \cdots, z_{n-1}\right\|\right)\right]^{p_{k}} \\
\leq & K \lim _{k \rightarrow \infty} u_{k}\left[M_{k}\left(\left\|\frac{\Delta_{m}^{n} x_{k}}{\rho_{1}}, z_{1}, \cdots, z_{n-1}\right\|\right)\right]^{p_{k}} \\
& +K \lim _{k \rightarrow \infty} u_{k}\left[M_{k}\left(\left\|\frac{\Delta_{m}^{n} y_{k}}{\rho_{2}}, z_{1}, \cdots, z_{n-1}\right\|\right)\right]^{p_{k}} \\
& =0 .
\end{aligned}
$$

So, $\alpha x+\beta y \in c_{0}\left(\mathcal{M}, \Delta_{m}^{n}, p, u,\|\cdot, \cdots, \cdot\|\right)$. Hence $c_{0}\left(\mathcal{M}, \Delta_{m}^{n}, p, u,\|\cdot, \cdots, \cdot\|\right)$ is a linear space. Similarly, we can prove that $c\left(\mathcal{N}, \Delta_{m}^{n}, p, u,\|\cdot, \cdots, \cdot\|\right)$ and $l_{\infty}\left(\mathcal{N}, \Delta_{m}^{n}, p, u, \| \cdot\right.$, $\cdots, \cdot \|)$ are linear spaces.

Theorem 2.2. Let $\mathcal{M}=\left(M_{k}\right)$ be a Musielak-Orlicz function, $p=\left(p_{k}\right)$ be a bounded sequence of positive real numbers and $u=\left(u_{k}\right)$ be a sequence of strictly positive real numbers. For $Z=l_{\infty}, c$ and $c_{0}$, the spaces $Z\left(\mathcal{N}, \Delta_{m}^{n}, p, u,\|\cdot, \cdots, \cdot\|\right)$ are paranormed spaces, paranormed by

$g(x)=\sum_{k=1}^{m n}\left\|x_{k}, z_{1}, \cdots, z_{n-1}\right\|+\inf \left\{\rho^{\frac{p_{k}}{H}}: \sup _{k} u_{k} M_{k}\left(\left\|\frac{\Delta_{m}^{n} x_{k}}{\rho}, z_{1}, \cdots, z_{n-1}\right\|\right) \leq 1\right\}$

where $H=\max \left(1, \sup _{k} p_{k}\right)$.

Proof. Clearly $g(-x)=g(x), g(\theta)=0$. Let $\left(x_{k}\right)$ and $\left(y_{k}\right)$ be any two sequences belong to any one of the space $Z\left(\mathcal{M}, \Delta_{m}^{n}, p, u,\|\cdot, \cdots, \cdot\|\right)$, for $Z=c_{0}, c$ and $l_{\infty}$. Then, we get $\rho_{1}, \rho_{2}>0$ such that

$$
\sup _{k} u_{k} M_{k}\left(\left\|\frac{\Delta_{m}^{n} x_{k}}{\rho_{1}}, z_{1}, \cdots, z_{n-1}\right\|\right) \leq 1
$$

and

$$
\sup _{k} u_{k} M_{k}\left(\left\|\frac{\Delta_{m}^{n} y_{k}}{\rho_{2}}, z_{1}, \cdots, z_{n-1}\right\|\right) \leq 1 .
$$

Let $\rho=\rho_{1}+\rho_{2}$. Then by convexity of $\mathcal{M}=\left(M_{k}\right)$, we have 


$$
\begin{aligned}
\sup _{k} u_{k} M_{k}\left(\left\|\frac{\Delta_{m}^{n}\left(x_{k}+y_{k}\right)}{\rho}, z_{1}, \cdots, z_{n-1}\right\|\right) \\
\leq\left(\frac{\rho_{1}}{\rho_{1}+\rho_{2}}\right) \sup _{k} u_{k} M_{k}\left(\left\|\frac{\Delta_{m}^{n} x_{k}}{\rho_{1}}, z_{1}, \cdots, z_{n-1}\right\|\right) \\
\quad+\left(\frac{\rho_{2}}{\rho_{1}+\rho_{2}}\right) \sup _{k} u_{k} M_{k}\left(\left\|\frac{\Delta_{m}^{n} y_{k}}{\rho_{2}}, z_{1}, \cdots, z_{n-1}\right\|\right) \\
\leq 1 .
\end{aligned}
$$

Hence we have,

$g(x+y)$

$$
\begin{aligned}
& =\sum_{k=1}^{m n}\left\|\left(x_{k}+y_{k}\right), z_{1}, \cdots, z_{n-1}\right\| \\
& +\inf \left\{\rho^{\frac{p_{k}}{H}}: \sup _{k} u_{k} M_{k}\left(\left\|\frac{\Delta_{m}^{n}\left(x_{k}+y_{k}\right)}{\rho}, z_{1}, \cdots, z_{n-1}\right\|\right) \leq 1\right\} \\
& \leq \sum_{k=1}^{m n}\left\|x_{k}, z_{1}, \cdots, z_{n-1}\right\|+\inf \left\{\rho_{1}^{\frac{p_{k}}{H}}: \sup _{k} u_{k} M_{k}\left(\left\|\frac{\Delta_{m}^{n} x_{k}}{\rho_{1}}, z_{1}, \cdots, z_{n-1}\right\|\right) \leq 1\right\} \\
& +\sum_{k=1}^{m n}\left\|y_{k}, z_{1}, \cdots, z_{n-1}\right\|+\inf \left\{\rho_{2}^{\frac{p_{k}}{H}}: \sup _{k} u_{k} M_{k}\left(\left\|\frac{\Delta_{m}^{n} y_{k}}{\rho_{2}}, z_{1}, \cdots, z_{n-1}\right\|\right) \leq 1\right\} .
\end{aligned}
$$

This implies that

$$
g(x+y) \leq g(x)+g(y)
$$

The continuity of the scalar multiplication follows from the following inequality

$g(\mu x)$

$$
\begin{aligned}
= & \sum_{k=1}^{m n}\left\|\mu x_{k}, z_{1}, \cdots, z_{n-1}\right\|+\inf \left\{\rho_{k}^{\frac{p_{k}}{H}}: \sup _{k} u_{k} M_{k}\left(\left\|\frac{\Delta_{m}^{n} \mu x_{k}}{\rho}, z_{1}, \cdots, z_{n-1}\right\|\right) \leq 1\right\} \\
= & |\mu| \sum_{k=1}^{m n}\left\|x_{k}, z_{1}, \cdots, z_{n-1}\right\| \\
& +\inf \left\{(t|\mu|)^{\frac{p_{k}}{H}}: \sup _{k} u_{k} M_{k}\left(\left\|\frac{\Delta_{m}^{n} x_{k}}{t}, z_{1}, \cdots, z_{n-1}\right\|\right) \leq 1\right\},
\end{aligned}
$$

where $t=\frac{\rho}{|\mu|}$. Hence the space $Z\left(\mathcal{M}, \Delta_{m}^{n}, p, u,\|\cdot, \cdots, \cdot\|\right)$, for $Z=c_{0}, c$ and $l_{\infty}$ is a paranormed space, paranormed by $g$.

Theorem 2.3. Let $\mathcal{M}=\left(M_{k}\right)$ be a Musielak-Orlicz function, $p=\left(p_{k}\right)$ be a bounded sequence of positive real numbers and $u=\left(u_{k}\right)$ be a sequence of strictly positive real numbers. For $Z=l_{\infty}, c$ and $c_{0}$, the spaces $Z\left(\mathcal{M}, \Delta_{m}^{n}, p, u,\|\cdot, \cdots, \cdot\|\right)$ are complete 
paranormed spaces, paranormed by

$g(x)=\sum_{k=1}^{m n}\left\|x_{k}, z_{1}, \cdots, z_{n-1}\right\|+\inf \left\{\rho^{\frac{p_{k}}{H}}: \sup _{k} u_{k} M_{k}\left(\left\|\frac{\Delta_{m}^{n} x_{k}}{\rho}, z_{1}, \cdots, z_{n-1}\right\|\right) \leq 1\right\}$,

where $H=\max \left(1, \sup _{k} p_{k}\right)$.

Proof. We prove the result for the space $l_{\infty}\left(\mathcal{M}, \Delta_{m}^{n}, p, u,\|\cdot, \cdots, \cdot\|\right)$. Let $\left(x^{i}\right)$ be any Cauchy sequence in $l_{\infty}\left(\mathcal{M}, \Delta_{m}^{n}, p, u,\|\cdot, \cdots, \cdot\|\right)$. Let $x_{0}>0$ be fixed and $t>0$ be such that for a given $0<\epsilon<1, \frac{\epsilon}{x_{0} t}>0$ and $x_{0} t \geq 1$. Then there exists a positive integer $n_{0}$ such that $g\left(x^{i}-x^{j}\right)<\frac{\epsilon}{x_{0} t}$, for all $i, j \geq n_{0}$. Using the definition of paranorm, we get

$$
\begin{aligned}
& \sum_{k=1}^{m n} \|\left(x_{k}^{i}-x_{k}^{j}, z_{1}, \cdots, z_{n-1} \|\right. \\
& \quad+\inf \left\{\rho^{\frac{p_{k}}{H}}: \sup _{k} u_{k} M_{k}\left(\left\|\frac{\Delta_{m}^{n}\left(x_{k}^{i}-x_{k}^{j}\right)}{\rho}, z_{1}, \cdots, z_{n-1}\right\|\right)\right\} \\
& <\frac{\epsilon}{x_{0} t}, \text { for all } i, j \geq n_{0} .
\end{aligned}
$$

Hence we have,

$$
\sum_{k=1}^{m n}\left\|\left(x_{k}^{i}-x_{k}^{j}\right), z_{1}, \cdots, z_{n-1}\right\|<\epsilon, \text { for all } i, j \geq n_{0} .
$$

This implies that

$$
\left\|\left(x_{k}^{i}-x_{k}^{j}\right), z_{1}, \cdots, z_{n-1}\right\|<\epsilon \text {, for all } i, j \geq n_{0} \text { and } 1 \leq k \leq m n .
$$

Thus $\left(x_{k}^{i}\right)$ is a Cauchy sequence for $k=1,2, \ldots ., m n$. Hence $\left(x_{k}^{i}\right)$ is convergent for $k=1,2, \ldots ., m n$. Let

$$
\lim _{i \rightarrow \infty} x_{k}^{i}=x_{k}, \quad \text { say for } k=1,2, \cdots, m n .
$$

Again from equation (2.1) we have,

$$
\inf \left\{\rho^{\frac{p_{k}}{H}}: \sup _{k} u_{k} M_{k}\left(\left\|\frac{\Delta_{m}^{n}\left(x_{k}^{i}-x_{k}^{j}\right)}{\rho}, z_{1}, \cdots, z_{n-1}\right\|\right) \leq 1\right\}<\epsilon \text {, for all } i, j \geq n_{0} .
$$

Hence we get

$$
\sup _{k} u_{k} M_{k}\left(\left\|\frac{\Delta_{m}^{n}\left(x_{k}^{i}-x_{k}^{j}\right)}{g\left(x^{i}-x^{j}\right)}, z_{1}, \cdots, z_{n-1}\right\|\right) \leq 1, \text { for all } i, j \geq n_{0} .
$$


It follows that $u_{k} M_{k}\left(\left\|\frac{\Delta_{m}^{n}\left(x_{k}^{i}-x_{k}^{j}\right)}{g\left(x^{i}-x^{j}\right)}, z_{1}, \cdots, z_{n-1}\right\|\right) \leq 1$, for each $k \geq 1$ and for all $i, j \geq n_{0}$. For $t>0$ with $u_{k} M_{k}\left(\frac{t x_{0}}{2}\right) \geq 1$, we have

$$
u_{k} M_{k}\left(\left\|\frac{\Delta_{m}^{n}\left(x_{k}^{i}-x_{k}^{j}\right)}{g\left(x^{i}-x^{j}\right)}, z_{1}, \cdots, z_{n-1}\right\|\right) \leq u_{k} M_{k}\left(\frac{t x_{0}}{2}\right) .
$$

This implies that

$$
\left\|\Delta_{m}^{n} x_{k}^{i}-\Delta_{m}^{n} x_{k}^{j}, z_{1}, \cdots, z_{n-1}\right\|<\frac{t x_{0}}{2} \frac{\epsilon}{t x_{0}}=\frac{\epsilon}{2}
$$

Hence $\left(\Delta_{m}^{n} x_{k}^{i}\right)$ is a Cauchy sequence for all $k \in \mathbb{N}$. This implies that $\left(\Delta_{m}^{n} x_{k}^{i}\right)$ is convergent for all $k \in \mathbb{N}$. Let $\lim _{i \rightarrow \infty} \Delta_{m}^{n} x_{k}^{i}=y_{k}$ for each $k \in \mathbb{N}$. Let $k=1$, then we have

$$
\lim _{i \rightarrow \infty} \Delta_{m}^{n} x_{1}^{i}=\lim _{i \rightarrow \infty} \sum_{v=0}^{n}(-1)^{v}\left(\begin{array}{c}
n \\
v
\end{array}\right) x_{1+m v}^{i}=y_{1} .
$$

We have by equation (2.2) and equation (2.3) $\lim _{i \rightarrow \infty} x_{m n+1}^{i}=x_{m n+1}$, exists. Proceeding in this way inductively, we have $\lim _{i \rightarrow \infty} x_{k}^{i}=x_{k}$ exists for each $k \in \mathbb{N}$. Now we have for all $i, j \geq n_{0}$,

$$
\begin{aligned}
& \sum_{k=1}^{m n}\left\|\left(x_{k}^{i}-x_{k}^{j}\right), z_{1}, \cdots, z_{n-1}\right\| \\
& \quad+\inf \left\{\rho^{\frac{p_{k}}{H}}: \sup _{k} u_{k} M_{k}\left(\left\|\frac{\Delta_{m}^{n}\left(x_{k}^{i}-x_{k}^{j}\right)}{\rho}, z_{1}, \cdots, z_{n-1}\right\|\right) \leq 1\right\}<\epsilon .
\end{aligned}
$$

This implies that

$$
\begin{aligned}
& \lim _{j \rightarrow \infty}\left\{\sum_{k=1}^{m n}\left\|\left(x_{k}^{i}-x_{k}^{j}\right), z_{1}, \cdots, z_{n-1}\right\|\right. \\
& \left.\quad+\inf \left\{\rho^{\frac{p_{k}}{H}}: \sup _{k} u_{k} M_{k}\left(\left\|\frac{\Delta_{m}^{n}\left(x_{k}^{i}-x_{k}^{j}\right)}{\rho}, z_{1}, \cdots, z_{n-1}\right\|\right) \leq 1\right\}\right\}<\epsilon,
\end{aligned}
$$

for all $i \geq n_{0}$. Using the continuity of $\left(M_{k}\right)$, we have

$$
\begin{aligned}
& \sum_{k=1}^{m n}\left\|\left(x_{k}^{i}-x_{k}\right), z_{1}, \cdots, z_{n-1}\right\| \\
& \quad+\inf \left\{\rho^{\frac{p_{k}}{H}}: \sup _{k} u_{k} M_{k}\left(\left\|\frac{\Delta_{m}^{n} x_{k}^{i}-\Delta_{m}^{n} x_{k}}{\rho}, z_{1}, \cdots, z_{n-1}\right\|\right) \leq 1\right\}<\epsilon,
\end{aligned}
$$

for all $i \geq n_{0}$. It follows that $\left(x^{i}-x\right) \in l_{\infty}\left(\mathcal{M}, \Delta_{m}^{n}, p, u,\|\cdot, \cdots, \cdot\|\right)$. Since $x^{i} \in l_{\infty}\left(\mathcal{M}, \Delta_{m}^{n}, p, u,\|\cdot, \cdots, \cdot\|\right)$ and $l_{\infty}\left(\mathcal{M}, \Delta_{m}^{n}, p, u,\|\cdot, \cdots, \cdot\|\right)$ is a linear space, so 
we have $x=x^{i}-\left(x^{i}-x\right) \in l_{\infty}\left(\mathcal{M}, \Delta_{m}^{n}, p, u,\|\cdot, \cdots, \cdot\|\right)$. This completes the proof. Similarly, we can prove that $c\left(\mathcal{M}, \Delta_{m}^{n}, p, u,\|\cdot, \cdots, \cdot\|\right)$ and $c_{0}\left(\mathcal{M}, \Delta_{m}^{n}, p, u,\|\cdot, \cdots, \cdot\|\right)$ are complete paranormed spaces in view of the above proof.

Theorem 2.4. If $0<p_{k} \leq q_{k}<\infty$ for each $k$, then $Z\left(\mathcal{M}, \Delta_{m}^{n}, p, u,\|\cdot, \cdots, \cdot\|\right) \subseteq$ $Z\left(\mathcal{M}, \Delta_{m}^{n}, q, u,\|\cdot, \cdots, \cdot\|\right)$, for $Z=c_{0}$ and $c$.

Proof. Let $x=\left(x_{k}\right) \in c\left(\mathcal{M}, \Delta_{m}^{n}, p, u,\|\cdot, \cdots, \cdot\|\right)$. Then there exists some $\rho>0$ and $L \in X$ such that

$$
\lim _{k \rightarrow \infty} u_{k}\left(M_{k}\left(\left\|\frac{\Delta_{m}^{n} x_{k}-L}{\rho}, z_{1}, \cdots, z_{n-1}\right\|\right)\right)^{p_{k}}=0 .
$$

This implies that $u_{k} M_{k}\left(\left\|\frac{\Delta_{m}^{n} x_{k}-L}{\rho}, z_{1}, \cdots, z_{n-1}\right\|\right)<\epsilon, \quad(0<\epsilon<1)$ for sufficiently large $k$. Hence we get

$$
\begin{aligned}
\lim _{k \rightarrow \infty} u_{k}\left(M_{k}\left(\left\|\frac{\Delta_{m}^{n} x_{k}-L}{\rho}, z_{1}, \cdots, z_{n-1}\right\|\right)\right)^{q_{k}} \\
\quad \leq \lim _{k \rightarrow \infty} u_{k}\left(M_{k}\left(\left\|\frac{\Delta_{m}^{n} x_{k}-L}{\rho}, z_{1}, \cdots, z_{n-1}\right\|\right)\right)^{p_{k}} \\
\quad=0 .
\end{aligned}
$$

This implies that $x=\left(x_{k}\right) \in c\left(\mathcal{M}, \Delta_{m}^{n}, q, u,\|\cdot, \cdots, \cdot\|\right)$. This completes the proof. Similarly, we can prove for the case $Z=c_{0}$.

Theorem 2.5. If $\mathcal{M}^{\prime}=\left(M_{k}^{\prime}\right)$ and $\mathcal{M}^{\prime \prime}=\left(M_{k}^{\prime \prime}\right)$ be two Musielak-Orlicz functions. Then

(i) $Z\left(\mathcal{M}^{\prime}, \Delta_{m}^{n}, p, u,\|\cdot, \cdots, \cdot\|\right) \subseteq Z\left(\mathcal{M}^{\prime \prime} \circ \mathcal{M}^{\prime}, \Delta_{m}^{n}, p, u,\|\cdot, \cdots, \cdot\|\right)$,

(ii) $Z\left(\mathcal{M}^{\prime}, \Delta_{m}^{n}, p, u,\|\cdot, \cdots, \cdot\|\right) \cap Z\left(\mathcal{M}^{\prime \prime}, \Delta_{m}^{n}, p, u,\|\cdot, \cdots, \cdot\|\right)$

$$
\subseteq Z\left(\mathcal{M}^{\prime}+\mathcal{M}^{\prime \prime}, \Delta_{m}^{n}, p, u,\|\cdot, \cdots, \cdot\|\right)
$$

for $Z=l_{\infty}, c$ and $c_{0}$.

Proof. (i) We prove this part for $Z=l_{\infty}$ and the rest of the cases will follow similarly. Let $\left(x_{k}\right) \in l_{\infty}\left(\mathcal{M}^{\prime}, \Delta_{m}^{n}, p, u,\|\cdot, \cdots, \cdot\|\right)$, then there exists $0<U<\infty$ such that

$$
u_{k}\left(M_{k}^{\prime}\left(\left\|\frac{\Delta_{m}^{n} x_{k}}{\rho}, z_{1}, \cdots, z_{n-1}\right\|\right)\right)^{p_{k}} \leq U, \text { for all } k \in \mathbb{N} .
$$

Let $y_{k}=u_{k} M_{k}^{\prime}\left(\left\|\frac{\Delta_{m}^{n} x_{k}}{\rho}, z_{1}, \cdots, z_{n-1}\right\|\right)$. Then $y_{k} \leq U^{\frac{1}{p_{k}}} \leq V$, say for all $k \in \mathbb{N}$.

Hence we have

$$
\left(\left(M_{k}^{\prime \prime} \circ M_{k}^{\prime}\right)\left(\left\|\frac{\Delta_{m}^{n} x_{k}}{\rho}, z_{1}, \cdots, z_{n-1}\right\|\right)\right)^{p_{k}}=\left(M_{k}^{\prime \prime}\left(y_{k}\right)\right)^{p_{k}} \leq\left(M_{k}^{\prime \prime}(V)\right)^{p_{k}}<\infty,
$$


for all $k \in \mathbb{N}$. Hence $\sup _{k} u_{k}\left(\left(M_{k}^{\prime \prime} \circ M_{k}^{\prime}\right)\left(\left\|\frac{\Delta_{m}^{n} x_{k}}{\rho}, z_{1}, \cdots, z_{n-1}\right\|\right)\right)^{p_{k}}<\infty$. Thus $x=\left(x_{k}\right) \in l_{\infty}\left(\mathcal{M}^{\prime \prime} \circ \mathcal{M}^{\prime}{ }^{k} \Delta_{m}^{n}, p, u,\|\cdot, \cdots, \cdot\|\right)$.

(ii) We prove the result for the case $Z=c$ and the rest of the cases will follow similarly. Let $x=\left(x_{k}\right) \in c\left(\mathcal{M}^{\prime}, \Delta_{m}^{n}, p, u,\|\cdot, \cdots, \cdot\|\right) \cap c\left(\mathcal{M}^{\prime \prime}, \Delta_{m}^{n}, p, u,\|\cdot, \cdots, \cdot\|\right)$, then there exist some $\rho_{1}, \rho_{2}>0$ and $L \in X$ such that

$$
\lim _{k \rightarrow \infty} u_{k}\left(M_{k}^{\prime}\left(\left\|\frac{\Delta_{m}^{n} x_{k}-L}{\rho_{1}}, z_{1}, \cdots, z_{n-1}\right\|\right)\right)^{p_{k}}=0
$$

and

$$
\lim _{k \rightarrow \infty} u_{k}\left(M_{k}^{\prime \prime}\left(\left\|\frac{\Delta_{m}^{n} x_{k}-L}{\rho_{2}}, z-1, \cdots, z_{n-1}\right\|\right)\right)^{p_{k}}=0 .
$$

Let $\rho=\rho_{1}+\rho_{2}$. Then we have

$$
\begin{aligned}
u_{k}\left(\left(M_{k}^{\prime}+M_{k}^{\prime \prime}\right)\right. & \left.\left(\left\|\frac{\Delta_{m}^{n} x_{k}-L}{\rho}, z_{1}, \cdots, z_{n-1}\right\|\right)\right)^{p_{k}} \\
\leq & K\left[\left(\frac{\rho_{1}}{\rho_{1}+\rho_{2}}\right) u_{k} M_{k}^{\prime}\left(\left\|\frac{\Delta_{m}^{n} x_{k}-L}{\rho_{1}}, z_{1}, \cdots, z_{n-1}\right\|\right)\right]^{p_{k}} \\
& +K\left[\left(\frac{\rho_{2}}{\rho_{1}+\rho_{2}}\right) u_{k} M_{k}^{\prime \prime}\left(\left\|\frac{\Delta_{m}^{n} x_{k}-L}{\rho_{2}}, z_{1}, \cdots, z_{n-1}\right\|\right)\right]^{p_{k}} .
\end{aligned}
$$

This implies that

$$
\lim _{k \rightarrow \infty} u_{k}\left(\left(M_{k}^{\prime}+M_{k}^{\prime \prime}\right)\left(\left\|\frac{\Delta_{m}^{n} x_{k}-L}{\rho}, z_{1}, \cdots, z_{n-1}\right\|\right)\right)^{p_{k}}=0 .
$$

Thus $x=\left(x_{k}\right) \in c\left(\mathcal{M}^{\prime}+\mathcal{M}^{\prime \prime}, \Delta_{m}^{n}, p, u,\|\cdot, \cdots, \cdot\|\right)$. This completes the proof.

Theorem 2.6. Let $\mathcal{M}=\left(M_{k}\right)$ be a Musielak-Orlicz function, $p=\left(p_{k}\right)$ be a bounded sequence of positive real numbers and $u=\left(u_{k}\right)$ be a sequence of strictly positive real numbers, then $Z\left(\mathcal{M}, \Delta_{m}^{n-1}, p, u,\|\cdot, \cdots, \cdot\|\right) \subset Z\left(\mathcal{M}, \Delta_{m}^{n}, p, u,\|\cdot, \cdots, \cdot\|\right)$, for $Z=$ $l_{\infty}, c$ and $c_{0}$.

Proof. We prove the result for the case $Z=l_{\infty}$ and the rest of the cases will follow similarly. Let $x=\left(x_{k}\right) \in l_{\infty}\left(\mathcal{M}, \Delta_{m}^{n-1}, p, u,\|\cdot, \cdots, \cdot\|\right)$. Then we can have $\rho>0$ such that

$$
u_{k}\left(M_{k}\left(\left\|\frac{\Delta_{m}^{n-1} x_{k}}{\rho}, z_{1}, \cdots, z_{n-1}\right\|\right)\right)^{p_{k}}<\infty, \quad \text { for all } k \in \mathbb{N} .
$$

On considering $2 \rho$ and using the convexity of $\left(M_{k}\right)$, we have

$$
\begin{aligned}
u_{k} M_{k}\left(\left\|\frac{\Delta_{m}^{n} x_{k}}{2 \rho}, z_{1}, \cdots, z_{n-1}\right\|\right) & \leq \frac{1}{2} u_{k} M_{k}\left(\left\|\frac{\Delta_{m}^{n-1} x_{k}}{\rho}, z_{1}, \cdots, z_{n-1}\right\|\right) \\
& +\frac{1}{2} u_{k} M_{k}\left(\left\|\frac{\Delta_{m}^{n-1} x_{k+m}}{\rho}, z_{1}, \cdots, z_{n-1}\right\|\right) .
\end{aligned}
$$


Hence we have

$$
\begin{aligned}
u_{k}\left(M_{k}\left(\left\|\frac{\Delta_{m}^{n} x_{k}}{2 \rho}, z_{1}, \cdots, z_{n-1}\right\|\right)\right)^{p_{k}} & \\
\leq & K\left\{u_{k}\left(\frac{1}{2} M_{k}\left(\left\|\frac{\Delta_{m}^{n-1} x_{k}}{\rho}, z_{1}, \cdots, z_{n-1}\right\|\right)\right)^{p_{k}}\right. \\
& \left.+u_{k}\left(\frac{1}{2} M_{k}\left(\left\|\frac{\Delta_{m}^{n-1} x_{k+m}}{\rho}, z_{1}, \cdots, z_{n-1}\right\|\right)\right)^{p_{k}}\right\} .
\end{aligned}
$$

Then using equation (2.4), we have

$$
u_{k}\left(M_{k}\left(\left\|\frac{\Delta_{m}^{n} x_{k}}{\rho}, z_{1}, \cdots, z_{n-1}\right\|\right)\right)^{p_{k}}<\infty, \text { for all } k \in \mathbb{N} .
$$

Thus $l_{\infty}\left(\mathcal{M}, \Delta_{m}^{n-1}, p, u,\|\cdot, \cdots, \cdot\|\right) \subset l_{\infty}\left(\mathcal{M}, \Delta_{m}^{n}, p, u,\|\cdot, \cdots, \cdot \cdot\|\right)$.

Theorem 2.7. Let $\mathcal{M}=\left(M_{k}\right)$ be a Musielak-Orlicz function. Then

$$
\begin{aligned}
c_{0}\left(\mathcal{M}, \Delta_{m}^{n}, p, u,\|\cdot, \cdots, \cdot\|\right) & \subset c\left(\mathcal{M}, \Delta_{m}^{n}, p, u,\|\cdot, \cdots, \cdot\|\right) \\
& \subset l_{\infty}\left(\mathcal{M}, \Delta_{m}^{n}, p, u,\|\cdot, \cdots, \cdot\|\right) .
\end{aligned}
$$

Proof. It is obvious that $c_{0}\left(\mathcal{M}, \Delta_{m}^{n}, p, u,\|\cdot, \cdots, \cdot\|\right) \subset c\left(\mathcal{M}, \Delta_{m}^{n}, p, u,\|\cdot, \cdots, \cdot\|\right)$. We shall prove that $c\left(\mathcal{M}, \Delta_{m}^{n}, p, u,\|\cdot, \cdots, \cdot\|\right) \subset l_{\infty}\left(\mathcal{M}, \Delta_{m}^{n}, p, u,\|\cdot, \cdots, \cdot\|\right)$. Let $x=\left(x_{k}\right) \in c\left(\mathcal{M}, \Delta_{m}^{n}, p, u,\|\cdot, \cdots, \cdot\|\right)$. Then there exists some $\rho>0$ and $L \in X$ such that

$$
\lim _{k \rightarrow \infty} u_{k}\left(M_{k}\left(\left\|\frac{\Delta_{m}^{n} x_{k}-L}{\rho}, z_{1}, \cdots, z_{n-1}\right\|\right)\right)^{p_{k}}=0 .
$$

On taking $\rho=2 \rho_{1}$, we have

$$
\begin{aligned}
& u_{k}\left(M_{k}\left(\| \frac{\Delta_{m}^{n} x_{k}}{\rho}, z_{1}, \cdots, z_{n-1}\right)\right)^{p_{k}} \\
& \leq K\left[\frac{1}{2} u_{k}\left(M_{k}\left(\left\|\frac{\Delta_{m}^{n} x_{k}-L}{\rho_{1}}, z_{1}, \cdots, z_{n-1}\right\|\right)\right)\right]^{p_{k}} \\
&+K\left[\frac{1}{2} u_{k} M_{k}\left(\left\|\frac{L}{\rho_{1}}, z_{1}, \cdots, z_{n-1}\right\|\right)\right]^{p_{k}} \\
& \leq K\left(\frac{1}{2}\right)^{p_{k}} u_{k}\left[M_{k}\left(\left\|\frac{\Delta_{m}^{n} x_{k}-L}{\rho_{1}}, z_{1}, \cdots, z_{n-1}\right\|\right)\right]^{p_{k}} \\
&+K\left(\frac{1}{2}\right)^{p_{k}} \max \left(1, u_{k}\left(M_{k}\left(\left\|\frac{L}{\rho_{1}}, z_{1}, \cdots, z_{n-1}\right\|\right)\right)^{H}\right),
\end{aligned}
$$

where $H=\max \left(1, \sup p_{k}\right)$. Thus we get $x=\left(x_{k}\right) \in l_{\infty}\left(\mathcal{M}, \Delta_{m}^{n}, p, u,\|\cdot, \cdots, \cdot\|\right)$. Hence $c_{0}\left(\mathcal{M}, \Delta_{m}^{n}, p, u,\|\cdot, \cdots, \cdot\|\right) \subset c\left(\mathcal{M}, \Delta_{m}^{n}, p, u,\|\cdot, \cdots, \cdot \cdot\|\right)$

$\subset l_{\infty}\left(\mathcal{M}, \Delta_{m}^{n}, p, u,\|\cdot, \cdots, \cdot\|\right)$. 
Theorem 2.8. The sequence space $l_{\infty}\left(\mathcal{M}, \Delta_{m}^{n}, p, u,\|\cdot, \cdots, \cdot\|\right)$ is solid.

Proof. Let $x=\left(x_{k}\right) \in l_{\infty}\left(\mathcal{M}, \Delta_{m}^{n}, p, u,\|\cdot, \cdots, \cdot\|\right)$, that is

$$
\lim _{k \rightarrow \infty} u_{k}\left[M_{k}\left(\left\|\frac{\Delta_{m}^{n} x_{k}}{\rho}, z_{1}, \cdots, z_{n-1}\right\|\right)\right]^{p_{k}}<\infty .
$$

Let $\left(\alpha_{k}\right)$ be a sequence of scalars such that $\left|\alpha_{k}\right| \leq 1$ for all $k \in \mathbb{N}$. Thus we have

$$
\begin{aligned}
\lim _{k \rightarrow \infty} u_{k}\left[M_{k}\left(\left\|\frac{\alpha_{k} \Delta_{m}^{n} x_{k}}{\rho}, z_{1}, \cdots, z_{n-1}\right\|\right)\right]^{p_{k}} \\
\leq \lim _{k \rightarrow \infty} u_{k}\left[M_{k}\left(\left\|\frac{\Delta_{m}^{n} x_{k}}{\rho}, z_{1}, \cdots, z_{n-1}\right\|\right)\right]^{p_{k}} \\
<\infty .
\end{aligned}
$$

This shows that $\left(\alpha_{k} x_{k}\right) \in l_{\infty}\left(\mathcal{M}, \Delta_{m}^{n}, p, u,\|\cdot, \cdots, \cdot\|\right)$ for all sequences of scalars $\left(\alpha_{k}\right)$ with $\left|\alpha_{k}\right| \leq 1$ for all $k \in \mathbb{N}$, whenever $\left(x_{k}\right) \in l_{\infty}\left(\mathcal{M}, \Delta_{m}^{n}, p, u, \| \cdot, \cdots, \cdot||\right)$. Hence the space $l_{\infty}\left(\mathcal{M}, \Delta_{m}^{n}, p, u,\|\cdot, \cdots, \cdot\|\right)$ is a solid sequence space.

Theorem 2.9. The sequence space $l_{\infty}\left(\mathcal{M}, \Delta_{m}^{n}, p, u,\|\cdot, \cdots, \cdot\|\right)$ is monotone.

Proof. The proof of the theorem is obvious and so we omit it.

Acknowledgement. The authors thank the referee for his valuable suggestions that improved the presentation of the paper.

\section{References}

[1] Y. Altın, Properties of some sets of sequences defined by a modulus function, Acta Math. Sci. Ser. B Engl. Ed., 29(2009), 427-434.

[2] M. Et, H. Altınok and Y. Altın, On generalized sequence spaces, Appl. Math. Comput., 154(2004), 167-173.

[3] M. Et, Y. Altın, B. Choudhary and B. C. Tripathy, On some classes of sequences defined by sequences of Orlicz functions, Math. Inequal. Appl., 9(2006), 335-342.

[4] M. Et and R. Çolak, On generalized difference sequence spaces, Soochow J. Math., 21(1995), 377-386.

[5] S. Gähler, Linear 2-normietre Rume, Math. Nachr., 28(1965), 1-43.

[6] H. Gunawan, On n-inner product, n-norms, and the Cauchy-Schwartz inequality, Sci. Math. Jap., 5(2001), 47-54.

[7] H. Gunawan, The space of p-summable sequence and its natural n-norm, Bull. Aust. Math. Soc., 64(2001), 137-147.

[8] H. Gunawan and M. Mashadi, On n-normed spaces, Int. J. Math. Math. Sci., 27(2001), 631-639.

[9] H. Kızmaz, On certain sequence spaces, Canad. Math-Bull., 24(1981), 169-176. 
[10] J. Lindenstrauss and L. Tzafriri, On Orlicz sequence spaces, Israel J. Math., 10(1971), 379-390.

[11] L. Maligranda, Orlicz spaces and interpolation, Seminars in Mathematics 5, Polish Academy of Science (1989).

[12] A. Misiak, n-inner product spaces, Math. Nachr., 140(1989), 299-319.

[13] J. Musielak, Orlicz spaces and modular spaces, Lecture Notes in Matthematics, 1034(1983).

[14] K. Raj, A. K. Sharma and S. K. Sharma, A Sequence space defined by a MusielakOrlicz function, Int. J. Pure Appl. Math., 67(2011), 475-484.

[15] K. Raj, S. K. Sharma and A. K. Sharma, Some difference sequence spaces in n-normed spaces defined by a Musielak-Orlicz function, Armen. J. Math., 3(2010), 127-141.

[16] K. Raj and S. K. Sharma, Some sequence spaces in 2-normed spaces defined by a Musielak-Orlicz function, Acta Univ. Sapientiae Math., 3(2011), 97-109.

[17] B. C. Tripathy and H. Dutta, Some difference paranormed sequence spaces defined by Orlicz functions, Fasciculi Math., Nr, 42(2009), 121-131.

[18] A. Wilansky, Summability through Functional Analysis, North- Holland Math. Stud., 85(1984). 